ISSN: 2639-0531

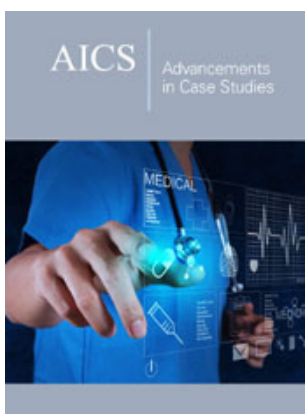

*Corresponding author: Nazia Hossain, Department of Internal Medicine, University of Toronto, Canada

Submission: 淟 December 03, 2020

Published: 鼓 December 10, 2020

Volume 2 - Issue 5

How to cite this article: Hossain $\mathrm{N}$, Eshtiaghi P, Miller Monthrope Y, Champagne T, Rashida Haq. DocetaxelInduced Supravenous Serpentine Dermatitis: A Case Report. Adv Case Stud. 2(5). AICS.000550. 2020

DOI: 10.31031/AICS.2020.02.000550

Copyright@ Hossain N, This article is distributed under the terms of the Creative Commons Attribution 4.0 International License, which permits unrestricted use and redistribution provided that the original author and source are credited.

\section{Docetaxel-Induced Supravenous Serpentine Dermatitis: A Case Report}

\author{
Hossain $\mathrm{N}^{1 *}$, Eshtiaghi $\mathrm{P}^{2}$, Miller Monthrope $\mathrm{Y}^{3}$, Champagne $\mathrm{T}^{2}$ and Rashida \\ $\mathrm{Haq}^{4}$ \\ ${ }^{1}$ Department of Internal Medicine, Canada \\ ${ }^{2}$ Department of Dermatology, Canada \\ ${ }^{3}$ Department of Laboratory Medicine, Canada \\ ${ }^{4}$ Department of Education-Oncology, Canada
}

\begin{abstract}
Background: Supra-venous serpentine dermatitis is a rare and self-limited cutaneous reaction to various chemotherapeutic agents.

Case presentation: We describe a case of supra-venous serpentine dermatitis triggered by docetaxel in a middle-aged female patient who received chemotherapy for triple negative breast cancer. Lesions affected the chest, neck, and the arm that was used to infuse docetaxel. The diagnosis was based on skin biopsy results showing pathology consistent with an interface dermatitis. The patient was treated with oral and topical glucocorticoids. Her second cycle of chemotherapy was infused at a slower rate, preceded and followed by boluses of normal saline. Her acute reaction resolved after 2 weeks and she tolerated her second cycle of docetaxel-cyclophosphamide without issues.
\end{abstract}

Conclusion: Oncologists and dermatologists should be aware of this potentially alarming reaction to docetaxel and other chemotherapeutic agents because adjustments to therapy are unnecessary and may result in undue harm as a result of suboptimal cancer treatment.

Keywords: Docetaxel; Supra-venous; Dermatitis; Cutaneous; Toxicity; Chemotherapy; Breast cancer

Abbreviations: ED: Emergency Department; HSV: Herpes Simplex Virus; PCR: Polymerase Chain Reaction; VZV: Varicella Zoster Virus

\section{Introduction}

Docetaxel is a taxane chemotherapy used to treat a variety of malignancies, including those of the breast and prostate [1,2]. It promotes stabilization of microtubules to inhibit cell division and promote apoptosis. Possible adverse effects can affect almost every organ including those of the cardiovascular, respiratory, gastrointestinal, nervous, and immune systems [3]. Cutaneous reactions are particularly common [4]. We describe a case of docetaxel-induced supra-venous serpentine dermatitis, which is a potentially alarming interface dermatitis with few case reports in the literature [5-11].

\section{Case Presentation}

A 61-year-old female presented to the emergency department (ED) with a one-day history of fever (temperature 38 degrees Celsius) and a two-day history of painless, nonpruritic vesicles on her right breast. Her past medical history was remarkable for a recent diagnosis of stage pT1cN0 triple negative breast cancer. She underwent lumpectomy and sentinel lymph node biopsy and had her first cycle of adjuvant chemotherapy with docetaxel and cyclophosphamide 5 days prior to her presentation to the ED. She had no other medical conditions and took no regular medications, vitamins, or supplements. She had no known drug allergies. Social and family histories were non-contributory. On dermatologic examination, she had two well-demarcated erythematous plaques studded with superficial papulovesicles on her right breast. She also had a number of small edematous and erythematous papules in a linear pattern on the central chest (Figure 1a). Swabs were collected for HSV/VZV PCR. She was initiated empirically on intravenous acyclovir. 
The next day, she developed similar lesions on her anterior and posterior neck (Figure 1b). In addition, she developed a cutaneous eruption on her left arm with an entirely different morphology from the vesicular lesions described. The eruption on her left arm was characterized by a dusky erythematous serpiginous plaque with prominent venous accentuation (Figure 1c). This was the same arm that had been used to infuse her chemotherapy one week prior. Over the next few days, the left arm eruption became more hypertrophic (Figure 1d). The patient also described marked pruritus in the area. All of the patient's cutaneous symptoms were managed conservatively with acetaminophen and diphenhydramine. HSV/ VZV PCR was negative, so acyclovir was discontinued. Blood and urine cultures also remained negative. A $3 \mathrm{~mm}$ punch biopsy was taken from the thickest part of the lesion on her arm. Pathology findings showed a lichenoid interface dermatitis with scattered eosinophils. Given the clinical context, the histological findings were consistent with docetaxel-induced supra-venous serpentine dermatitis.

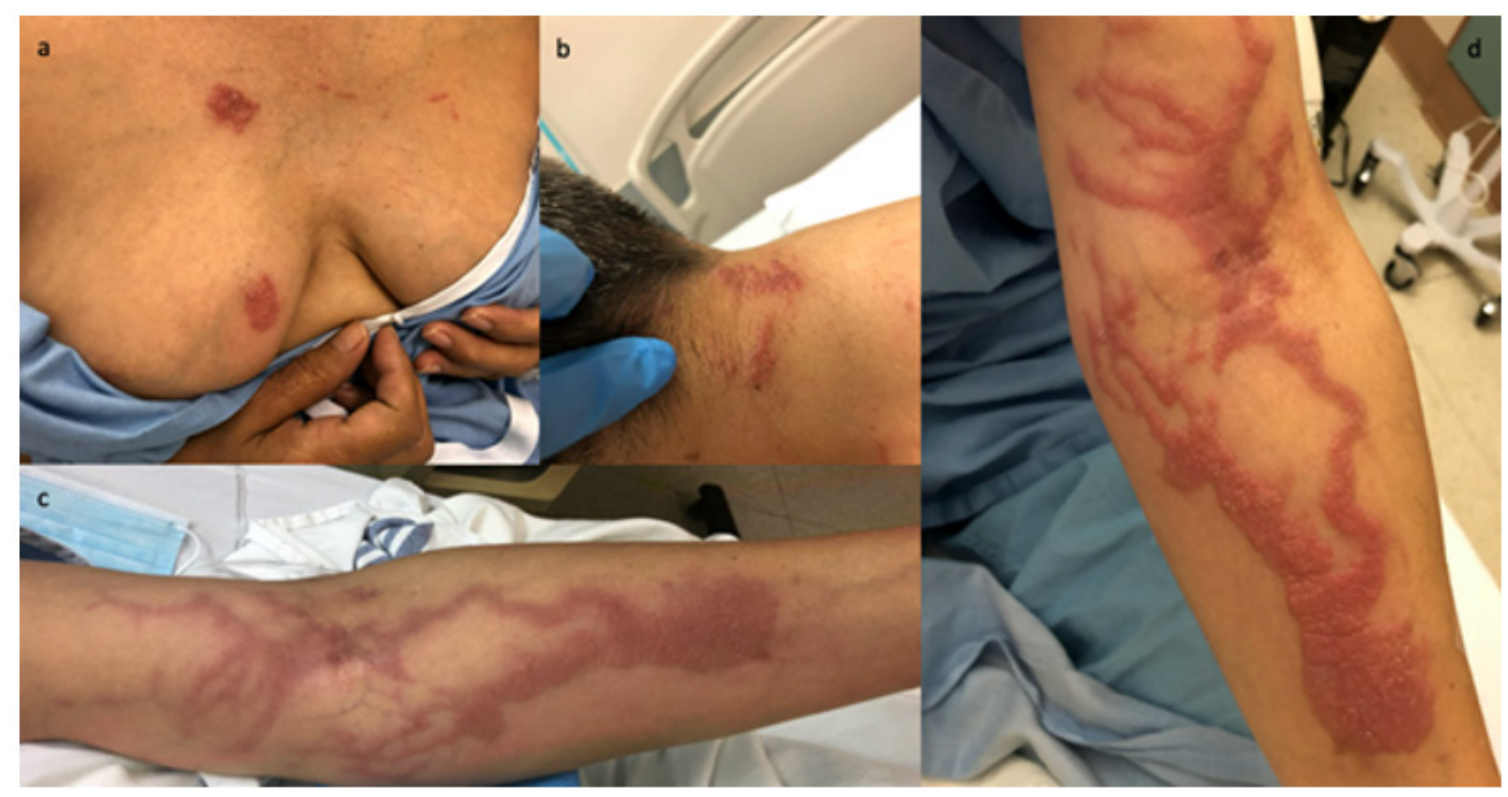

Figure 1: (a) Day 1-Erythematous plaques and papulovesicles on right breast (b) Day 2-Eruption on posterior neck (c) Day 2-Dusky erythematous eruption with prominent venous accentuation on left arm (d) Days 3 and 4-Left arm eruption became more hypertrophic

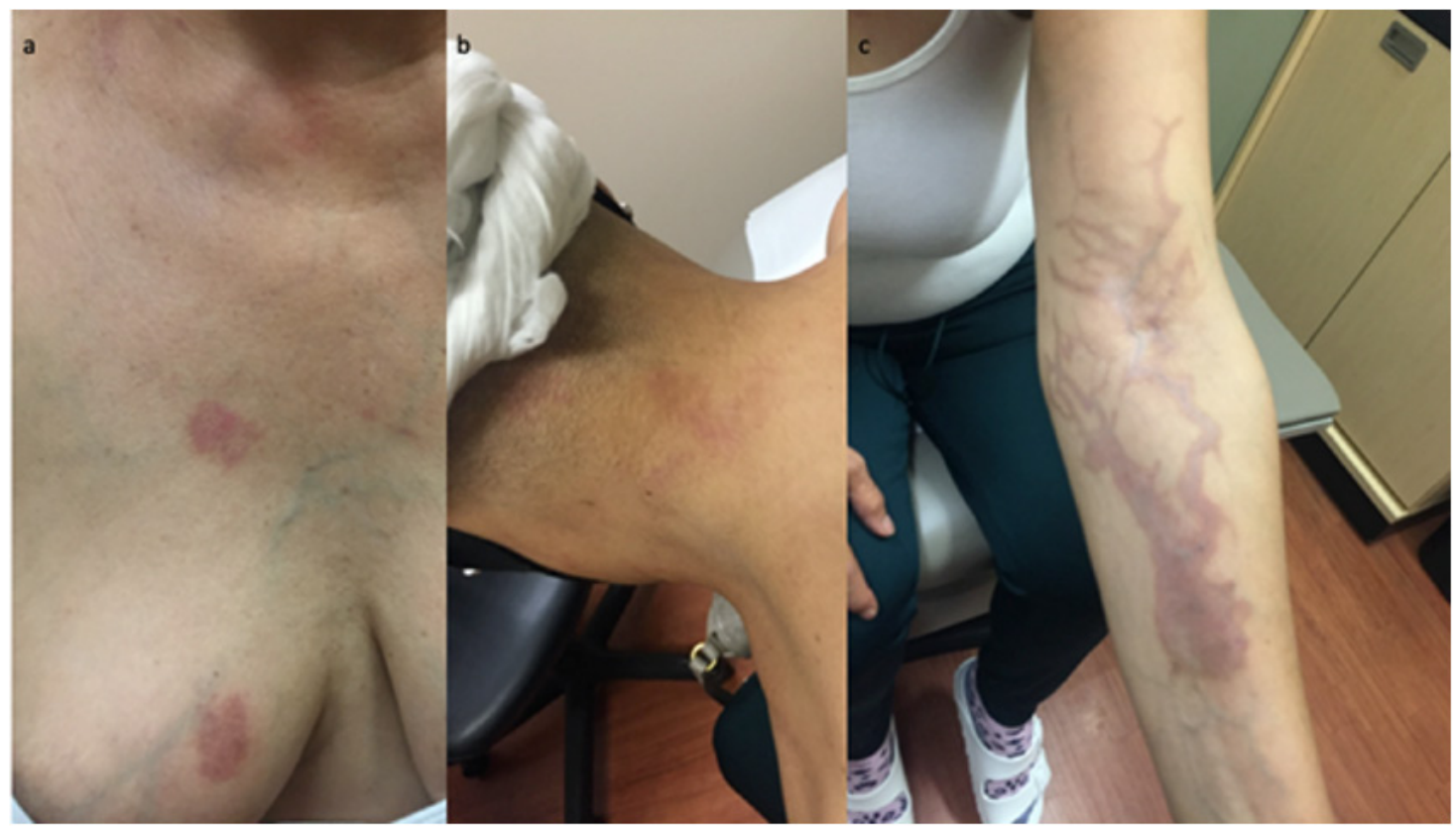

Figure 2: (a) Lesions on breast (b) neck and (c) left arm after treatment with oral prednisone for 7 days and topical betamethasone for 14 days. 
The patient was discharged home with a prescription for a seven-day course of oral prednisone $20 \mathrm{mg}$, as well as betamethasone valerate $0.1 \%$ cream applied to the affected areas twice daily for 14 days. After two weeks, she was seen in follow-up by her medical oncologist for consideration of her second cycle of docetaxel and cyclophosphamide. Her lesions had improved remarkably (Figure 2). Her second cycle of chemotherapy was infused at a slower rate and she was administered $250 \mathrm{~mL}$ of intravenous normal saline before, as well as $500 \mathrm{~mL}$ after, her chemotherapy. She was also given a three-day prescription for oral dexamethasone $2 \mathrm{mg}$ twice daily to be taken after her chemotherapy. She tolerated her second cycle well with no adverse events. Despite the resolution of her initial eruption, post inflammatory pigmentation remains in the areas affected. This is a finding seen following many inflammatory conditions including almost all interface dermatitides, especially in skin of coloured patients [12]. Damage to the basal cell layer of the epidermis where melanocytes are present leads to melanin pigment being released into the dermis. The resulting hyperpigmentation may take years to resolve completely. Avoidance of ultraviolet radiation and regular use of sunscreen can help to speed recovery.

\section{Discussion}
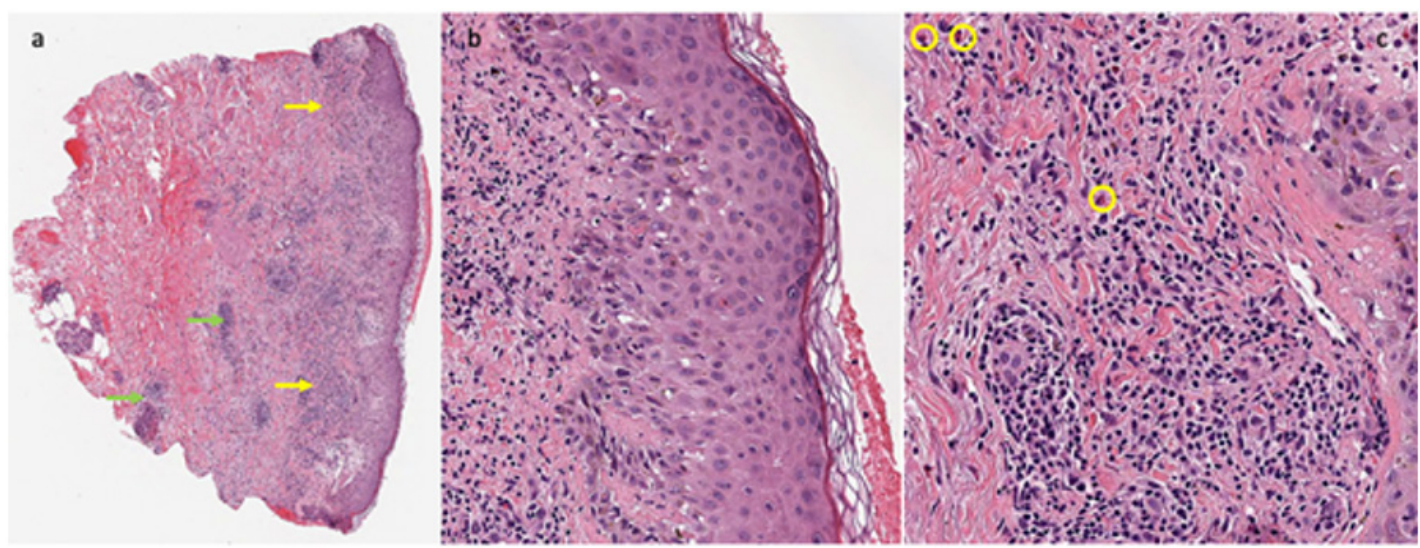

Figure 3: (a) This low power view highlights irregular acanthosis, and a band like inflammatory cell infiltrate abutting the dermal epidermal junction (yellow arrows). This view also highlights the deep perivascular and periadnexal inflammatory infiltrates (green arrows) (b) This image shows vacuolar degeneration, numerous necrotic keratinocytes and scattered melanophages (indicating damage to the basal layer) (c) This high-power image highlights rare eosinophils within the inflammatory infiltrate which is predominantly lymphocytes (yellow circles).

Supra-venous serpentine dermatitis is a rare cutaneous adverse reaction to irritant medications or chemicals that are infused intravenously. Few case reports are available in the literature of similar phenomena caused by chemotherapeutic agents such as carboplatin, vinorelbine [5], docetaxel [6-9] and anti-tumour necrosis factor-alpha [10]. Lesions are described as erythematous macules or purpuric papules that typically develop 24 hours to 15 days after exposure. The exact mechanism of toxicity remains unclear. It is hypothesized that irritant chemicals cause direct cytotoxic injury to the vascular endothelium and, in turn, the dermal and epidermal layers as well as keratinocytes and melanocytes. Pathological findings are generally reported to show hyperkeratosis, acanthosis, focal spongiosis, necrotic keratinocytes, melano-phages, and an interface dermatitis with marked vacuolar degeneration of the basal cell layer. The presence of a perivascular inflammatory infiltrate extending to the deep dermis has also been reported $[6,10]$ (Figure 3).

Supravenous serpentine dermatitis is a self-limited reaction and typically resolves in several weeks to months. However, patients may have residual hyperpigmentation of the affected skin. The acute eruption can be managed with a medium-potency topical steroid with or without oral glucocorticoids. Importantly, there is no indication to discontinue or decrease the dose of the offending agent [5-7]. In order to prevent the reaction with subsequent exposures, intravenous medications should be infused at a slower rate with boluses of normal saline before and after to wash out any toxic metabolites from blood vessels. During long infusion sessions, central access is preferred over peripheral access. Cold compresses can be applied over the site of infusion to cause venous constriction and degradation of metabolites. In addition, compresses can be used during the acute reaction to decrease pain and inflammation [5-7].

\section{Conclusion}

Supravenous serpentine dermatitis is a rare cutaneous drug eruption secondary to irritant intravenous medications. The acute reaction is managed with topical and oral glucocorticoids. There is no indication to discontinue or adjust the dose of the offending agent. Preventative strategies include using slower infusion rates and infusing boluses of normal saline before and after the drug. Knowledge of this adverse event and its management allowed our patient to continue first line therapy for her malignancy.

\section{Acknowledgement}

All clinical images were used with permission granted by the patient. 


\section{References}

1. Lyseng-Williamson KA, Fenton C (2005) Docetaxel: A review of its use in metastatic breast cancer. Drugs 65(17): 2513-2531.

2. Basch E, Loblaw DA, Oliver TK, Carducci M, Chen RC, et al. (2014) Systemic therapy in men with metastatic castration-resistant prostate cancer: American Society of Clinical Oncology and Cancer Care Ontario clinical practice guideline. J Clin Oncol 32(30): 3436-3448.

3. Ho MY, Mackey JR (2014) Presentation and management of docetaxelrelated adverse effects in patients with breast cancer. Cancer Manag and Res 6: 253-259.

4. Sibaud V, Loboeuf NR, Roche H, Belum VR, Gladieff L, et al. (2016) Dermatological adverse events with taxane chemotherapy. Eur J Dermatol 26(5): 427-443.

5. Akyurek FT, Sari N, Ugurluoglu C, Kurtipek GS (2019) Serpentine supravenous hyperpigmentation related to carboplatin and vinorelbine chemotherapy: A case report. Dermatol Ther 32(4): e12981.

6. Aydogan I, Kavak A, Parlak AH, Alper M, Annakkaya AN, et al. (2005) Persistent serpentine supravenous hyperpigmented eruption associated with docetaxel. J Eur Acad Dermatol Venereol 19(3): 345-347.
7. Kamei H, Yano T, Kiura K, Umemura S, Yamane H, et al. (2009) A case of persistent serpentine supravenous hyperpigmented eruption associated with docetaxel. Lung Cancer 49(2): 198-201.

8. Chaiyakul S (2018) Docetaxel-induced supravenous serpentine dermatitis: A case report and literature review. J Med Assoc Thai 101: 1297-301.

9. Fernandes KAP, Felix PAO (2015) Persistent docetaxel-induced supravenous erythematous eruption. An Bras Dermatol 90(5): 728-730.

10. Garcovich S, Burlando M, Rongioletti F, Garcovich A, Parodi A, et al (2009) Cutaneous drug eruption with an interface dermatitis pattern due to anti-tumour necrosis factor-alpha agents: A relevant class-effect. Acta Dermatol Venereol 90(3): 311-312.

11. Spencer HJ (1984) Local erythema multiforme-like drug reaction following intravenous mitomycin C and 5-fluorouracil. J Surg Oncol 26(1): 47-50.

12. Davis EC, Callender VD (2010) Post-inflammatory hyperpigmentation: A review of the epidemiology, clinical features, and treatment options in skin of color. J Clin Aesthet Dermatol 3(7): 20-31. 